Original

\title{
Dietary D-psicose, a Rare Sugar, Shows No Modifying Effects in a Medium-term Liver Carcinogenesis Bioassay in F344 Male Rats
}

\author{
Yu Zeng ${ }^{1}$, Kousuke Saoo ${ }^{1}$, Masanao Yokohira ${ }^{1}$, Hijiri Takeuchi ${ }^{1}$, Jia-Qing Li $^{1}$, \\ Keiko Yamakawa $^{1}$, Yoko Matsuda ${ }^{1}$, and Katsumi Imaida ${ }^{1}$ \\ ${ }^{1}$ Onco-Pathology, Department of Pathology and Host-Defense, Faculty of Medicine, Kagawa University, 1750-1 \\ Ikenobe, Miki-cho, Kita-gun, Kagawa 761-0793, Japan
}

\begin{abstract}
D-psicose, a C-3 epimer of D-fructose, is present in very small quantities in commercial carbohydrate complexes and agricultural products, and is therefore called a rare sugar. The effects of D-psicose on diethylnitrosamine (DEN)-induced hepatocarcinogenesis were examined in male F344 rats using a rat medium-term bioassay based on the two-step model of hepatocarcinogenesis. The modifying potential was determined by comparing the numbers and areas/ $\mathrm{cm}^{2}$ of induced glutathione $S$-transferase placental form (GST-P) positive foci in the liver with those of a corresponding group (control) of rats given DEN alone. Increased relative liver weights were found in the $1 \%$ D-psicose treatment group as compared with the basal diet group, while no significant change occurred in the $0.1 \% \mathrm{D}$-psicose, $0.01 \% \mathrm{D}$ psicose, and $1 \%$ D-fructose groups. D-psicose did not significantly alter the numbers and area/ $\mathrm{cm}^{2}$ of GST-P positive liver cell foci observed after DEN initiation. The results thus demonstrate that D-psicose shows neither promoting nor preventive potential for liver carcinogenesis in our medium-term bioassay. (J Toxicol Pathol 2005; 18: 85-88)
\end{abstract}

Key words: D-psicose, rare sugar, hepatocarcinogenesis, GST-P-positive liver cell foci, rat

\section{Introduction}

D-psicose (D-ribo-2-hexulose), a C-3 epimer of Dfructose, is present in small quantities in commercial mixtures of D-glucose and D-fructose, which is obtained from hydrolysis of sucrose or isomerization of D-glucose ${ }^{1-3}$. According to its extremely low quantities naturally occurring, D-psicose is included in a group of isomers of monosaccharides, known as rare sugars. In the past few decades, some isomers of monosaccharides, for example, Lsugar or D-tagatose, have been developed as alternative carbohydrate sweeteners and bulking agents ${ }^{4,5}$ as sugar substitutes, because of the disadvantages of sucrose intake, namely, lipogenic potency ${ }^{6,7}$ and high insulinogenicity ${ }^{8}$. Such sweeteners typically have an energy content approximately one-half of that of sucrose, $16.5 \mathrm{~kJ} / \mathrm{g}^{9}$. Supplement of D-psicose in the diet has been found to suppress hepatic lipogenic enzyme activities in rats compared with supplements of D-fructose or D-glucose and

Received: 7 January 2005, Accepted: 10 March 2005

Mailing address: Katsumi Imaida, Onco-Pathology, Department of

Pathology and Host-Defense, Faculty of Medicine, Kagawa

University, 1750-1 Ikenobe, Miki-cho, Kita-gun, Kagawa 761-0793, Japan

TEL: 81-87-891-2109 FAX: 81-87-891-2112

E-mail:imaida@med.kagawa-u.ac.jp provide zero energy ${ }^{10}$. However, more intensive study about this rare sugar has been impeded by the difficulty in preparing it by chemical methods. Recently, an improved method $^{11}$ has been developed which is applicable to the large-scale production of D-psicose from D-fructose, and it should enable the commercial utilization of this presently expensive sugar. D-psicose might, therefore, be useful in the food industry as a non-calorie sweetener for obese people as an aid for weight reduction. Further understanding the properties of this rare sugar, including its modifying potential in humans, appears to be necessary.

The aim of the present study was to investigate the carcinogenic or preventive potential of D-psicose on diethylnitrosamine (DEN)-initiated hepatocarcinogenesis in F344 male rats. A medium-term liver carcinogenesis bioassay (Ito test) was employed in this study. This 8 -week liver bioassay system has been developed on the basis of initiation-promotion theory for carcinogenesis which proposes that genotoxic carcinogens have both initiating and promoting activities ${ }^{12-14}$. The liver medium-term bioassay is a very useful tool for the detection of hepatocarcinogenicity, and is appropriate for the assessment of low dose effects because of its high sensitivity ${ }^{15-18}$. Glutathione $S$-transferase placental form (GST-P) positive foci, which can be readily detected by immunohistochemistry, are established preneoplastic lesions, and are used as end-points to assess 
the carcinogenic or preventive response.

\section{Materials and Methods}

\section{Chemicals}

D-psicose (lot 0112114, 2,059) and D-fructose (lot $111 \mathrm{k} 0055)$ were obtained from Kagawa Rare Sugar Cluster (Kagawa, Japan). DEN was purchased from Tokyo Kasei Kogyo Co. Ltd. (Tokyo, Japan).

\section{Animals}

A total of 78, 4-week-old, male F344 rats were obtained from Charles River Japan Inc. (Atsugi, Japan). The animals were housed in an air-conditioned room at a temperature of $22 \pm 1{ }^{\circ} \mathrm{C}$ and a relative humidity of $60 \pm 10 \%$, with a $12 \mathrm{~h}$ light/dark cycle, and maintained on an basal pellet diet (Oriental MF, Oriental Yeast Co., Tokyo, Japan) and tap water ad libitum. They were used in the experiment after a two-week acclimation period. This animal study was conducted in accordance with the laws and related operation rules on the treatment and use of laboratory animals.

\section{Treatment of animals}

The experiment protocol for the medium-term liver bioassay is shown in Fig. $1^{19}$. The animals were randomly divided into five groups, with 18 rats in group 4 and 15 rats in each of the other groups. All groups were given a single intraperitoneal injection of DEN (200 mg/kg body weight) dissolved in $0.9 \% \mathrm{NaCl}$ solution to initiate hepatocarcinogenesis on the first day of the experiment. After 2 weeks on basal diet, they were administered basal diet containing D-psicose at doses of $1.0 \%, 0.1 \%, 0.01 \%$, and 0 (control), or $1.0 \% \mathrm{D}$-fructose for the following 6 weeks. D-fructose was used here as another control because D-psicose is synthesized from it and they share a similar chemical structure. All the rats were subjected to two-thirds partial hepatectomy $(\mathrm{PH})$ at the end of week 3. Body weights and food consumption were recorded every two weeks and all surviving rats were sacrificed under ether anesthesia at the end of week 8 of the experiment.

\section{Tissue processing}

At autopsy, livers were immediately excised, weighed and fixed in $10 \%$ neutral buffered formalin. Kidneys on both sides were excised and fixed at the same time. They were used to evaluate the common toxic effect of the compounds. After $48 \mathrm{~h}$ fixing, livers were cut into 2- to 3-mm-thick slices, one each from the right and caudate lobes and two from the right anterior lobe. The slices of liver and kidney were embedded in paraffin for routine histopathological examination and immunohistochemical staining of GST-P.

\section{Immunohistochemistry}

To determine the location of GST-P binding in the liver, immunohistochemical staining was performed using the VENTANA HX system (VENTANA, Yokohama, Japan). Liver sections were treated with rabbit anti-rat GST-P

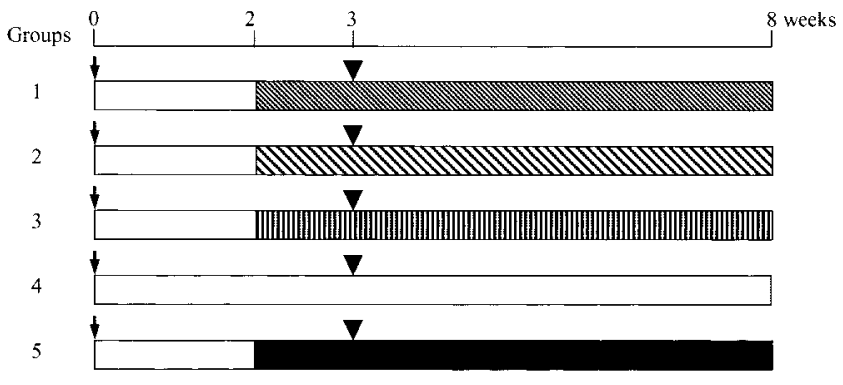

Fig. 1. Experimental protocol for assessment of effects of D-psicose on DEN-induced hepatocarcinogenesis in a medium-term liver bioassay. Animals, 6-week-old F344 male rats; $\downarrow$, diethylnitrosamine (DEN) $200 \mathrm{mg} / \mathrm{kg}$ b.w., i.p.; $\boldsymbol{\nabla}$, twothirds partial hepatectomy; $1 \%$ D-psicose in diet; $0.1 \%$ D-psicose in diet; $\mid$ |l|l|l, $0.01 \%$ D-psicose in diet; $\square$, basal diet; $\square, 1 \%$ D-fructose in diet.

antibody (MBL, Nagoya, Japan, 1:2000) and then sequentially with secondary antibody and the avidin-biotin complex $(\mathrm{ABC})$ reaction. The numbers and areas of GST-P positive foci with diameters larger than $0.2 \mathrm{~mm}$ and the total areas of the livers were measured using an Image Processor for Analytical Pathology (IPAP-WIN, Sumika Technos Co., Osaka, Japan $)^{20}$. The numbers and areas of foci $/ \mathrm{cm}^{2}$ of liver section were calculated.

\section{Statistical analysis}

The inter-group differences in body weights, organ weights and GST-P positive liver foci numbers or areas $/ \mathrm{cm}^{2}$ were assessed using analysis of variance (ANOVA). When positive results were obtained by ANOVA, Dunnett's multiple comparison test was applied to evaluate the statistical significance between treatment groups. $P$ values less than 0.05 were considered significant.

\section{Results}

Two rats in the group 1, two in the group 2, and one each in the groups 4 and 5 were found dead on the day of partial hepatectomy. The cause of death was considered to be incomplete ligation. Totally, 72 rats were included in the final data. Body weights and relative liver weights are shown in Table 1. There were no significant differences for the final body, or the absolute or relative kidney weights among groups. The absolute and relative liver weights of rats given $1 \%$ D-psicose (group 1) were significantly increased compared with that of the respective control (group 4). The food consumption did not significantly differ among groups, and the intake of the target component in the diet varied as expected.

There were no macroscopic or microscopic abnormalities observed for either the liver or kidney in the treated groups. Foci of cellular alteration could be identified even in routine hematoxylin and eosin (H\&E) staining sections. Hepatic foci varied in size from small foci 
Table 1. Final Body and Liver Weights, and D-psicose/D-fructose Intake

\begin{tabular}{clccccc}
\hline \multirow{2}{*}{ Group } & \multirow{2}{*}{ Treatment } & \multirow{2}{*}{ Effective No. of rats } & Body weight $(\mathrm{g})$ & \multicolumn{2}{c}{ Liver weight } & \multirow{2}{*}{$\begin{array}{c}\text { Total D-psicose/D-fructose } \\
\text { intake (mg/rat) }\end{array}$} \\
& & & Absolute (g) & Relative (\%) & & 6.3319 \\
2 & $1.0 \%$ D-psicose & 13 & $232.2 \pm 10.6$ & $8.2 \pm 0.4^{*}$ & $3.5 \pm 0.2^{* *}$ & 0.6861 \\
3 & $0.1 \%$ D-psicose & 13 & $234.7 \pm 7.2$ & $7.5 \pm 0.6$ & $3.2 \pm 0.3$ & 0.0613 \\
4 & Basal D-psicose & 15 & $236.2 \pm 9.1$ & $7.6 \pm 0.5$ & $3.2 \pm 0.2$ & 0 \\
5 & $1.0 \%$ D-fructose & 14 & $235.9 \pm 8.4$ & $7.6 \pm 0.7$ & $3.2 \pm 0.3$ & 6.5867 \\
\hline
\end{tabular}

*, **: Significantly different from the basal diet group value at $P<0.05, P<0.01$, respectively.

Table 2. Quantitative Results for GST-P Positive Liver Cell Foci

\begin{tabular}{clccc}
\hline \multirow{2}{*}{ Group } & Treatment & Effective No. of rats & \multicolumn{2}{c}{ GST-P positive liver cell foci } \\
\cline { 4 - 5 } & & & Area $\left(\mathrm{mm}^{2} / \mathrm{cm}^{2}\right)$ & Number $\left(\mathrm{No} . / \mathrm{cm}^{2}\right)$ \\
\hline 1 & $1.0 \%$ D-psicose & 13 & $0.21 \pm 0.12$ & $2.5 \pm 1.0$ \\
2 & $0.1 \%$ D-psicose & 13 & $0.21 \pm 0.10$ & $2.8 \pm 0.8$ \\
3 & $0.01 \%$ D-psicose & 15 & $0.25 \pm 0.09$ & $3.3 \pm 0.6$ \\
4 & Basal diet & 17 & $0.21 \pm 0.10$ & $2.9 \pm 1.3$ \\
5 & $1.0 \%$ D-fructose & 14 & $0.18 \pm 0.12$ & $2.6 \pm 1.6$ \\
\hline
\end{tabular}

comprising several altered hepatocytes to large ones occupying an area equivalent to several adjacent lobules. Foci were usually of the clear cell type, being composed of enlarged cells with rarefied clear cytoplasm and central nuclei. Foci were not associated with any compression of the surrounding parenchyma. Quantitative data for GST-P positive foci per $\mathrm{cm}^{2}$ of liver tissue are summarized in Table 2. The numbers of GST-P positive hepatocytic foci were inclined to decrease in rats treated with D-psicose in a doserelated manner, however, this tendency was not significant. Furthermore, no significant difference for the areas of GST$P$ positive foci per $\mathrm{cm}^{2}$ was found between any treatment group and the control group.

\section{Discussion}

D-psicose has ever been considered as a sugar substitute because it provides zero energy ${ }^{10}$. Recently, with the development of new production methods, more and more studies focus on this previously scarce monosaccharide ${ }^{21}$. As well as suppressing hepatic lipogenic enzyme activities ${ }^{10}$, D-psicose has also been found to reduce hyperlipidemia. Additionally, D-psicose inhibits D-fructose being absorbed by the intestines, and promotes insulin secretion of pancreas $\beta$ cells in rats; therefore, it shows therapeutic effects for insulin-dependent diabetes (unpublished data from the Kagawa Rare Sugar Cluster). However, there is limited knowledge about the modifying effects of this rare sugar on carcinogenesis.

The medium-term rat liver bioassay employed in the present study for the detection of carcinogens has been validated with more than 300 chemical substances ${ }^{22}$, and focal GST-P expression has proved to be the most accurate marker for rat preneoplastic foci ${ }^{23}$. This system has become widely recognized as a bridge to overcome the disadvantages of both in vitro short-term screening tests and long-term in vivo bioassays, by allowing accuracy at a reasonable cost in terms of both time and money ${ }^{24}$. It has been demonstrated that chemicals which give positive results in this system are hepatocarcinogens or hepatopromoting agents in the long term $^{25,26}$. Also, compounds that inhibit the development of GST-P positive foci in this system are generally regarded as effective in chemoprevention ${ }^{22}$. In the present study, in the histological evaluation of the liver, there were no significant differences in the numbers of preneoplastic lesions in rats fed with D-psicose at a range of doses compared with control values. The numbers and areas of GST-P positive foci in the rat livers were not affected by the intake of D-psicose for the 8 -week experimental term. Although the numbers of GST-P positive foci in rats fed D-psicose decreased in a dose-related manner, the results were not significant. The present results demonstrate that D-psicose has neither preventing nor promoting effects on DEN-initiated rat liver carcinogenesis. In line with the present results, it was shown that another rare sugar D-allose, but not D-psicose, inhibited cell proliferation in some human cancer cell lines (unpublished data from the Kagawa Rare Sugar Cluster). The modifying potential of other rare sugars should be explored in future studies.

In the present study, the absolute and relative liver weights were increased in the highest D-psicose intake group as compared with the basal diet group. The reason for this is unclear. Intake of D-psicose has been found to stimulate insulin secretion (unpublished data from the Kagawa Rare Sugar Cluster), and insulin sequentially promotes glycogen synthesis in the liver, It is possible that this mechanism may have led to the increase of liver weights seen in this study; however, we did not observe hypertrophy in liver cells in this group. Further study is clearly needed to clarify the 
mechanism of increased liver weight.

In summary, the data obtained from the present investigation suggest that D-psicose possesses neither hepatocarcinogenicity nor chemoprevention effects in F344 male rats. It should be a valuable work for exploring the further utilization of this rare sugar.

Acknowledgements: This work was supported by a research grant from the Cooperative Link of Unique Science and Technology for Economy Revitalization (CLUSTER).

\section{Reference}

1. Cree GM and Perlin AS. O-isopropylidene derivatives of Dallulose (D-psicose) and D-erythro-hexopyranos-2,3diulose. Can J Biochem. 46: 765-770. 1968.

2. Binkley WW. The fate of cane juice simple sugars during molasses formation IV. Probable conversion of D-fructose to D-psicose. Int Sugar J. 65: 105-106. 1963.

3. Miller BS and Swain T. Chromatographic analyses of the free amion acids, organic acids and sugars in wheat plant extracts. J Sci Food Agric. 11: 344-348. 1969.

4. Giffhorn F, Kopper S, Huwig A, and Freimund S. Rare sugars and sugar-based synthons by chemo-enzymatic synthesis. Enzyme Microb Technol. 27: 734-742. 2000.

5. Manzoni M, Rollini M, and Bergomi S. Biotransformation of D-galactitol to tagatose by acetic acid bacteria. Process Biochem. 36: 971-977. 2001.

6. Campell RG and Zinner DD. Effect of certain dietary sugars on hamster carries. J Nutr. 100: 10-20. 1970.

7. Ooshima T, Izumitani A, Sobue S, Okahashi N, and Hamada S. Non-cariogenicity of the disaccharide palatinose in experimental dental caries of rats. Infect Immun. 39: 43-49. 1983.

8. Kawai K, Okuda Y, and Yamashita K. Changes in blood glucose and insulin after an oral palatinose administration in normal subjects. Endocrinol Jpn. 32: 933-936. 1985.

9. Levin GV, Zehner LR, Saunders JP, and Beadle JR. Sugar substitutes: their energy values, bulk characteristics, and potential health benefits. Am J Clin Nutr. 62: 1161S-1168S. 1995.

10. Matsuo T, Baba Y, Hashiguchi M, Takeshita K, Izumori K, and Suzuki H. Dietary D-psicose, a C-3 epimer of Dfructose, suppresses the activity of hepatic lipogenic enzymes in rats. Asia Pacific J Clin Nutr. 10: 233-237. 2001.

11. Takeshita K, Suga A, Takada G, and Izumori K. Mass production of D-psicose from $\mathrm{D}$-fructose by a contimuous bioreactor system using immobilized D-tagatose 3epimerase. J Biosci Bioeng. 90: 453-455. 2000.

12. Tatematsu $M$, Hasegawa $R$, Imaida $K$, Tsuda $H$, and Ito $N$. Survey of various chemicals for initiating and promoting activities in a short-term in vivo system based on generation of hyperplastic liver nodules in rats. Carcinogenesis. 4: 381386. 1983.

13. Hasegawa R, Futakuchi M, Mizoguchi Y, Yamaguchi T,
Shirai T, Ito N, and Lijinsky W. Studies of initiation and promotion of carcinogenesis by N-nitroso compounds. Cancer Lett. 123: 185-191. 1998.

14. Ito N, Tatematsu M, Nakanishi K, Hasegawa R, Takano T, Imaida $\mathrm{K}$, and Ogiso $\mathrm{T}$. The effects of various chemicals on the development of hyperplastic liver nodules in hepatectomized rats treated with $\mathrm{N}$-nitrosodiethylamine or N-2-fluorenylacetamide. Gann. 71: 832-842. 1980.

15. Sakai H, Tsukamoto $T$, Yamamoto $M$, Shirai $N$, Iidaka $T$, Yanai T, Masegi T, and Tatematsu M. Differential effects of partial hepatectomy and carbon tetrachloride administration on induction of liver cell foci in a model for detection of initiation activity. Jpn J Cancer Res. 92: 1018-1025. 2001.

16. Tsuda H, Lee $G$, and Farber E. Induction of resistant hepatocytes as a new principle for a possible short-term in vivo test for carcinogens. Cancer Res. 40: 1157-1164. 1980.

17. Ito N, Tsuda H, Tatematsu M, Inoue T, Tagawa Y, Aoki T, Uwagawa S, Kagawa M, Ogiso T, Masui T, Imaida K, Fukushima S, and Asamoto M. Enhancing effect of various hepatocarcinogens on induction of preneoplastic glutathione S-transferase placental form positive foci in rats--an approach for a new medium-term bioassay system. Carcinogenesis. 9: 387-394. 1988.

18. Ito $N$, Hasegawa $R$, Imaida $K$, Hirose $M$, and Shirai $T$. Medium-term liver and multi-organ carcinogenesis bioassays for carcinogens and chemopreventive agents. Exp Toxicol Pathol. 48: 113-119. 1996.

19. Shirai T. A medium-term rat liver bioassay as a rapid in vivo test for carcinogenic potential: a historical review of model development and summary of results from 291 tests. Toxicol Pathol. 25: 453-460. 1997.

20. Watanabe T, Katsura A, and Yoshitake A. Image processors for analytical pathology. J Toxicol Pathol. 7: 353-361. 1994.

21. Sun Y, Hayakawa S, and Izumori K. Modification of ovalbumin with a rare ketohexose through the Maillard reaction: effect on protein structure and gel properties. J Agric Food Chem. 52: 1293-1299. 2004.

22. Ito N, Imaida K, Asamoto M, and Shirai T. Early detection of carcinogenic substances and modifiers in rats. Mutat Res. 462: 209-217. 2000.

23. Hendrich S, Campbell HA, and Pitot HC. Quantitative stereological evaluation of four histochemical markers of altered foci in multistage hepatocarcinogenesis in the rat. Carcinogenesis. 8: 1245-1250. 1987.

24. Ito N, Hasegawa R, Imaida K, Hirose M, and Shirai T. Medium-term rat liver bioassay for rapid detection of hepatocarcinogenic substances. J Toxicol Pathol. 10: 1-11. 1997.

25. Ito N, Hasegawa R, Imaida $K$, Takahashi $S$, and Shirai $T$. Medium-term rat liver bioassay for rapid detection of carcinogens and modifiers of hepatocarcinogenesis. Drug Metab Rev. 26: 431-442. 1994.

26. Ogiso $\mathrm{T}$, Tatematsu M, Tamano $\mathrm{S}$, Hasegawa R, and Ito $\mathrm{N}$. Correlation between medium-term liver bioassay system data and results of long-term testing in rats. Carcinogenesis. 11: 561-566. 1990. 